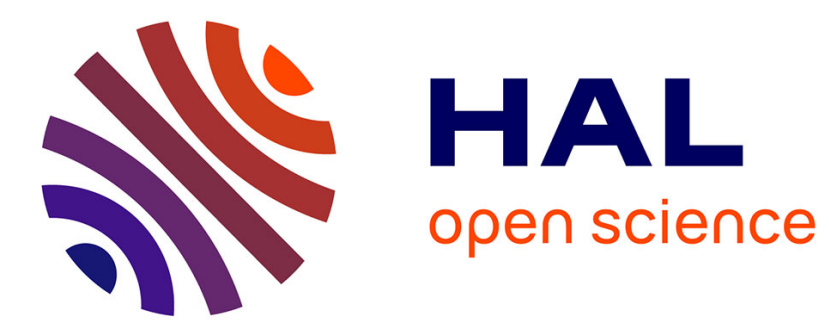

\title{
Patterning in systems driven by nonlocal external forces
}

\author{
Laurence Luneville, K Mallick, V Pontikis, D Simeone
}

\section{To cite this version:}

Laurence Luneville, K Mallick, V Pontikis, D Simeone. Patterning in systems driven by nonlocal external forces. Physical Review E , 2016, 94, pp.052126. 10.1103/PhysRevE.94.052126 . hal01496968

\section{HAL Id: hal-01496968 \\ https://hal.science/hal-01496968}

Submitted on 28 Mar 2017

HAL is a multi-disciplinary open access archive for the deposit and dissemination of scientific research documents, whether they are published or not. The documents may come from teaching and research institutions in France or abroad, or from public or private research centers.
L'archive ouverte pluridisciplinaire HAL, est destinée au dépôt et à la diffusion de documents scientifiques de niveau recherche, publiés ou non, émanant des établissements d'enseignement et de recherche français ou étrangers, des laboratoires publics ou privés. 


\title{
Patterning in systems driven by nonlocal external forces
}

\author{
L. Luneville, ${ }^{1}$ K. Mallick, ${ }^{2}$ V. Pontikis, ${ }^{3}$ and D. Simeone ${ }^{4}$ \\ ${ }^{1}$ DEN-Service dÉtudes et de Recherche en Mathématique Appliquée, LRC CARMEN CEA-CNRS-ECP/SPMS, \\ Université Paris-Saclay, F-91191, Gif-sur-Yvette, France \\ ${ }^{2}$ CEA/DRF/IPhT, CEA, Université Paris-Saclay, F-91191, Gif-sur-Yvette, France, \\ ${ }^{3}$ CEA/DRF/IRAMIS/LSI, CEA, Université Paris-Saclay, F-91191, Gif-sur-Yvette, France, \\ ${ }^{4}$ DEN-Service de Recherches Métallurgiques Appliquées, LRC CARMEN CEA-CNRS-ECP/SPMS, \\ Université Paris-Saclay, F-91191, Gif-sur-Yvette, France
}

(Received 22 April 2016; revised manuscript received 7 August 2016; published 16 November 2016)

\begin{abstract}
This work focuses on systems displaying domain patterns resulting from competing external and internal dynamics. To this end, we introduce a Lyapunov functional capable of describing the steady states of systems subject to external forces, by adding nonlocal terms to the Landau Ginzburg free energy of the system. Thereby, we extend the existing methodology treating long-range order interactions, to the case of external nonlocal forces. By studying the quadratic term of this Lyapunov functional, we compute the phase diagram in the temperature versus external field and we determine all possible modulated phases (domain patterns) as a function of the external forces and the temperature. Finally, we investigate patterning in chemical reactive mixtures and binary mixtures under irradiation, and we show that the last case opens the path toward micro-structural engineering of materials.
\end{abstract}

DOI: 10.1103/PhysRevE.94.052126

\section{INTRODUCTION}

Several physical and chemical systems exhibit macroscopic patterning $[1,2]$, ranging in diversity from superconducting films to reaction-diffusion (Turing) mixtures and to liquids forming Rayleigh-Bénard instabilities [3]. At equilibrium, domain shapes and patterns, which can be understood as spatially modulated phases, are computed from the minimization of a free-energy functional [3,4]. However, no generally accepted theoretical framework exists applying to dissipative systems driven by external forces $[5,6]$, whereas numerical simulations operating at the atomic scale are unable to predict the experimentally observed macroscopic patterns [7].

Heterogeneous systems forming under the action of thermodynamic forces (phase transformations) or mechanical instabilities [2] have been studied thanks to the Landau-Ginzburg (LG) free-energy functional. Moreover, the dynamics of diffusional transformations is well described by the CahnHilliard $(\mathrm{CH})$ evolution equation $[2,3,8]$. In this framework, the mean-field description of an evolving system is made in terms of the relevant conserved order parameter $\eta(\mathbf{x}, t)$, representing by example the atomic density [4], the atomic composition [2], or the local deviation of the monomer density from its average value [8]. On the other hand, the LG free energy $F(\eta(\mathbf{x}, t))$ describes short-range atomic interactions, splitting into bulk and interfacial contributions to the free energy. Both terms are functions of the order parameter and its derivatives $[2,4]$ :

$$
F(\eta(\mathbf{x}, t))=\int\left[f(\eta(\mathbf{x}, t))+\frac{\kappa}{2}|\nabla \eta(\mathbf{x}, t)|^{2}\right] d \mathbf{x},
$$

where $f(\eta(\mathbf{x}, t))$ is the free-energy density, the parameters of which are generally fitted to satisfactorily reproduce equilibrium phases and $\kappa$ is related to the energetic cost of emerging interfaces. Nucleation and growth of heterogeneous phases during the evolution toward equilibrium are then obtained solving the $\mathrm{CH}$ equation:

$$
\frac{\partial \eta(\mathbf{x}, t)}{\partial t}=M \nabla^{2}\left[\frac{\delta F(\eta(\mathbf{x}, t))}{\delta \eta(\mathbf{x}, t)}\right]
$$

where $M$ is the mobility of species assumed to be order parameter independent [9] and the fluctuations of the order parameter are neglected $[1,10]$. Elastic or coulombic nonlocal interactions deriving from an effective pairwise interaction potential, $W\left(x-x^{\prime}\right)$, can be additively introduced in $F(\eta(\mathbf{x}, t))$ [8,11], as shown by Leibler [12], and Ohta and Kawasaki [8] for the case of block copolymers at equilibrium. For an evolution governed by the $\mathrm{CH}$ equation, the nature, the number, and the energy barriers associated with the equilibrium phases depend only on the extrema of $F(\eta(\mathbf{x}, t))$. Besides, previous works clearly show that the existence of ordered and disordered phases at thermodynamic equilibrium can be determined solely from the study of the quadratic term of this free-energy functional $[1,2,8,9,13]$.

In this work, the LG functional and the $\mathrm{CH}$ evolution equation are used for predicting microstructures forming in systems driven by nonlocal external forces within the classical phase field theory framework. Two distinct methods have been used for determining ordered states and their limits, the linear stability analysis and the study of the structure factor [14]. The linear stability analysis can only be performed in the vicinity of the steady states, which requires numerically minimizing the kinetic equation governing the evolution of the system driven by non local external forces. An alternative to this numerical approach consists in computing the structure factor and estimating the modulation wave vector $\mathbf{k}_{\mathbf{0}}$ associated with steady states $[15,16]$. However, this last method is computationally expensive and is based on crude approximations, restricted to the $O(N)$ model describing only second-order phase transition [17] and constraining the form of $f(\eta(\mathbf{x}, t))$ [15]. The present work, though proceeding by analogy with the work by Ohta et al. [8], differs from it and other studies [14-16,18], as here 
the forces acting on the considered system are external and cannot be only related with a competition between shortand long-range order interactions and does not involve any assumptions about the symmetry of $f(\eta(\mathbf{x}, t))$. Moreover, we show that the number, the modulation of ordered steady states, and their limits emerge from the analysis of the quadratic term of a Lyapounov functional, without explicitly solving the kinetic equation describing the competition between different dynamics.

\section{DIFFERENT NONLOCAL EXTERNAL FORCES}

In the following, to predict the microstructure in systems driven by nonlocal forces, focus is made on systems whose evolution involves the competition between the phase separation and the disordering induced by nonlocal external forces $[2,8]$.

\section{A. Diblock copolymers}

When two incompatible polymer chains $A_{i}$ and $B_{j}$ of lengths $i$ and $j$ are mixed, their evolution toward equilibrium leads to a phase separation into two coexisting phases. When these chains are covalently bounded at their ends, a diblock copolymer $A_{i} B_{j}$ forms and phase separation cannot occur. This interaction can be modeled within the phase field framework assuming an effective force field conjugated to the order parameter $\eta(\mathbf{x}, t)$ expressed as the average deviation from the uniform distribution of monomers $A$ and $B$ [12]. This effect is accounted for by adding a new dynamic to the $\mathrm{CH}$ equation describing the phase separation:

$$
\left(\frac{\partial \eta(\mathbf{x}, t)}{\partial t}\right)^{\mathrm{ext}}=-\Gamma \eta(\mathbf{x}, t)
$$

where $\Gamma$ related with the effective force field is a frequency defining the time scale of this dynamics.

\section{B. Chemically reactive mixtures}

For binary reactive mixtures, the demixing via a spinodal decomposition is counterbalanced by a chemical reaction between two components $A \underset{\Gamma_{2}}{\stackrel{\Gamma_{1}}{\rightleftarrows}} B$, where $\Gamma_{j}$ are the reaction rate constants associated with the chemical reaction. Alike the case of diblock copolymers, the system evolution is dictated by an external force as

$$
\left(\frac{\partial \eta(\mathbf{x}, t)}{\partial t}\right)^{\mathrm{ext}}=-\Gamma \eta(\mathbf{x}, t)
$$

where $\Gamma$ is the sum of the reaction rate constants and $\eta(\mathbf{x}, t)$ is related to the local variation of the A concentration. Such an equation can also be derived directly from a master equation based on the Ising model on lattice gas Hamiltonian with reaction [19].

\section{Immiscible alloys under irradiation}

The phase field approach has been also used for describing the behavior of alloys with positive heat of mixing under irradiation [16]. The positive heat of mixing insures that a spinodal decomposition occurs in these alloys out of irradiation. On the other hand, The slowing down of particles in the alloys under irradiation tends to spatially mix atoms regardless of their chemical identity. This process counterbalances the spinodal decomposition driving the system into steady states [18]. For alloys under irradiation, $\Gamma$ is a function of the mass, the energy, the flux of impinging particles, and the density of the target. The ejection of atoms from their equilibrium position can be modeled with a probability density function $p_{R}(x) \propto$ $\exp \left(-\frac{|x|}{R}\right)$, where $R$ is associated with a characteristic length scale [20]. The external dynamics can be written as

$$
\left(\frac{\partial \eta(\mathbf{x}, t)}{\partial t}\right)^{\mathrm{ext}}=-\Gamma\left[\int p_{R}(\mathbf{x}-\mathbf{y}) \eta(\mathbf{y}) d \mathbf{y}-\eta(\mathbf{x}, t)\right],
$$

where $\eta(\mathbf{x}, t)$ is equal to the difference between the atomic fractions of the two components. In this description, $\Gamma$ and $R$ define the characteristic time and length scales of the problem.

Assuming the characteristic length scale of the problem is infinite, i.e., $R \rightarrow \infty$, Eqs. (3) and (4) are recovered from Eq. (5). This last equation including characteristic length and time scales can be understood as a master equation describing the action of external non local forces in diffusive processes. Within this framework, $\Gamma p_{R}(x)$ is the transition rate for the external dynamics. Equation (5) then appears to be quite general and can be applied to a large class of dissipative systems.

\section{PATTERNING FORMATION}

Within the phase field theory framework, the $\mathrm{CH}$ equation describes the formation of mesoscopic domains in the long wave limit and Eq. (5) is formally similar to an effective nonlocal singular pairwise interaction potential describing long-range interactions. In the present approach, the competition between two dynamics is dictated by the ratio $\frac{\Gamma}{M}$. The steady states resulting from this competition then depend explicitly from this ratio, i.e., the mobility. This is the main difference between this work and previous extensions of the phase field approach applied to describe mesoscopic patterns produced at equilibrium in systems submitted to nonlocal forces.

\section{A. Lyapounov functional}

The mathematical expression describing the competition between the spinodal decomposition and dynamics driven by external forces, $\left(\frac{\partial \eta(\mathbf{x}, t)}{\partial t}\right)^{\text {ext }}$ for these dissipative systems can be rewritten as

$$
\frac{\partial \eta(\mathbf{x}, t)}{\partial t}=M \nabla^{2}\left[\frac{\delta F(\eta(\mathbf{x}, t))}{\delta \eta(\mathbf{x}, t)}\right]+\left(\frac{\partial \eta(\mathbf{x}, t)}{\partial t}\right)^{\mathrm{ext}} .
$$

This equation expresses the competition between different dynamics that act in parallel. Because dynamics acts at different time scale, the balance between these two dynamics creates unexpected ordered steady states, which do not appear in the equilibrium phase diagram. Including the external dynamics due to nonlocal interactions in the $\mathrm{CH}$ equation, a Lyapounov functional can be calculated $[8,11]$ :

$$
\frac{\partial \eta(\mathbf{x}, t)}{\partial t}=M \nabla^{2}\left[\frac{\delta \mathcal{L}(\eta(\mathbf{x}, t))}{\delta \eta(\mathbf{x}, t)}\right]
$$


with

$$
\mathcal{L}(t)=F(\eta(\mathbf{x}, t))+\frac{\Gamma}{2 M} \iint \eta(\mathbf{x}, t) g(\mathbf{x}-\mathbf{y}) \eta(\mathbf{y}, t) d \mathbf{x} d \mathbf{y},
$$

where $g(\mathbf{x})$ is the Green function for the Laplace equation, $\nabla^{2} g(\mathbf{x})=p_{R}(\mathbf{x})-\delta(\mathbf{x})$, and accounts for external forces. It can be shown that $\mathcal{L}(t)$ is a decreasing function of time with a lower bound, i.e., it is a Lyapounov functional [21] defining the global stability of this dissipative system. The freeenergy functional $F(\eta(\mathbf{x}, t))$ is now replaced by a Lyapunov functional in Eq. (7), describing dynamics of dissipative systems satisfying Eq. (5). The dynamics behavior of systems described by Eq. (7) consists in relaxation toward the minimum of $\mathcal{L}(t)$ leading to the formation of patterns associated with ordered steady states. Since $\mathcal{L}(t)$ is a Lyapunov functional, no steady states associated with limit cycles nor chaotic evolution of the order parameter can emerge in these systems [1].

It should also be noticed that no faithful analogy can be established with this approach and the usual way of including long-range interaction to the short-range order in the Landau expansion at equilibrium $[8,11]$. In this last approach, the Lyapunov does not contain any information on the time scale. In our present approach, the characteristic time scale associated with the two dynamics $\frac{\Gamma}{M}$ highlights the impact of two dynamics on the pattern formation. Steady states resulting from the minimization of Eq. (8) are then not independent of the different dynamics as it is the case at the equilibrium. When $\Gamma$ and $M$ differ by orders of magnitude, there is no balance between competitive dynamical phenomena leading therefore either to demixion or to a homogeneous disordered state. When $\Gamma \approx M$, the two dynamics compete and unexpected modulated steady states could emerge.

\section{B. Existence of steady states}

As in the equilibrium case [8,11], the analysis of the quadratic term of the Lyapunov functional characterizes the force-driven steady states. By introducing explicitly the temperature in $\mathcal{L}(t)$, it becomes possible to compute a phase diagram describing the number and the nature of steady states as a function of the control parameters and the temperature.

Going one step further requires defining explicitly the free-energy functional. A choice compatible with the case studies explored in this work is the Landau free-energy functional known to successfully apply to binary mixture [2]. The Landau free energy is based on a mean-field approximation. Therefore, this approximation does not introduce any substantial errors since mesoscopic phases are associated with the long wave limit in the phase field approach. Improvement of the free-energy model was to only change the Landau expansion coefficients changing the dimension of the mesoscopic structures without affecting their appearance. The second-order coefficient of the Landau-Ginzburg expansion can be written as $a_{2}(T)=-a T_{c} \tau$ where $T_{c}$ is the absolute instability temperature of the system in the absence of external forces [2], $\tau=\frac{T_{c}-T}{T_{c}}$ is the reduced temperature, and $a$ is a positive constant [4].
Introducing characteristic length and time scales $x^{\prime}=\frac{x}{l_{0}}$, $t^{\prime}=\frac{t}{t_{0}}$, with $l_{0}=\sqrt{\frac{\kappa \tau}{a_{4} \alpha^{2}}}, t_{0}=\frac{\kappa \tau^{2}}{M a_{4}^{2} \Omega \alpha^{4}}, \Omega$ the atomic volume, $\eta_{ \pm}$the values of the order parameter associated with the minima of the homogeneous free energy density, and $\alpha=\frac{\eta_{+}-\eta_{-}}{2}$, the Lyapounov functional takes the following dimensionless form (reduced units are used hereafter with the superscript "prime" dropped for sake of simplicity):

$$
\begin{aligned}
\mathcal{L}(t)= & \int\left[-\frac{\tau\left(1-3 \lambda^{2}\right)}{2} \psi^{2}-\sqrt{\tau} \lambda \psi^{3}+\frac{\psi^{4}}{4}+|\nabla \psi|^{2}\right] d \mathbf{x} \\
& +\frac{W}{2} \iint \psi(\mathbf{y}, t) g(\mathbf{x}-\mathbf{y}) \psi(\mathbf{x}, t) d \mathbf{x} d \mathbf{y}
\end{aligned}
$$

where $W=t_{0} \Gamma$ is the reduced exchange frequency and is directly related to different dynamics in competition, $\lambda=$ $\frac{\eta_{+}+\eta_{-}}{\eta_{+}-\eta_{-}}$and $\psi(\mathbf{x}, t)=\eta(\mathbf{x}, t) \frac{\sqrt{\tau}}{\alpha}$ is the reduced order parameter.

The quadratic term of the Lyapunov functional can be written in the Fourier space as

$$
\hat{b}(k, \tau)=-\tau\left(1-3 \lambda^{2}\right)+k^{2}+W \hat{g}(k) .
$$

Note that the contribution of the external force to the behavior of the system is entirely contained in $\hat{b}(k, \tau)$ and is characterized by the amplitude $W$.

When all the $\hat{b}(k, \tau)$ are positive, the disordered steady state is stable with respect to infinitesimal fluctuations and, conversely, it becomes unstable upon $\hat{b}(k, \tau)$ reaching negative values. A necessary condition for $\hat{b}(k, \tau)$ to reach its minimum at $k=k_{0}$ is given by

$$
\frac{\partial \hat{b}}{\partial k}\left(k_{0}(\tau), \tau\right)=0 .
$$

When $\hat{b}\left(k_{0}(\tau), \tau\right)$ is positive, only the disordered phase exists. When $\hat{b}\left(k_{0}(\tau), \tau\right)$ is negative, an ordered phase with a modulation wave vector $k_{0}(\tau)$ emerges. The number of wave vectors $k_{0}$ solution of Eq. (11) determines the number of different ordered steady states. On the other hand, the absolute instability temperature $T_{c}^{W}=T_{c}\left(1-\tau_{c}^{W}\right)$ [22] defines the temperature range associated with these ordered steady states, with $\tau_{c}^{W}$ solution of

$$
\hat{b}\left(k_{0}\left(\tau_{c}^{W}\right), \tau_{c}^{W}\right)=0 .
$$

From this implicit equation, the limit between the ordered and disordered steady states can be drawn as a function of the control parameters describing external forces and temperature. This limit is different from the one obtained from the linear stability analysis of Eq. (7) which involves $k^{2} \hat{b}$ instead of $\hat{b}$ [18]. This limit and the wave vectors $k_{0}$ of the ordered phases result from the instability of $\mathcal{L}(t \rightarrow \infty)$ and only depend on Eq. (7), i.e., the dynamics of the problem, via $\hat{g}(k)$.

\section{APPLICATIONS OF THE METHOD}

The above consideration provides a procedure effective for predicting the occurrence of steady states under external forces and their domains of existence at different temperatures, thus bypassing the complexity of calculating the values of the order parameter. In the following, first is re-examined the case of a diblock copolymer subject to long-range order forces by calculating its equilibrium phase diagram, and retrieving the 

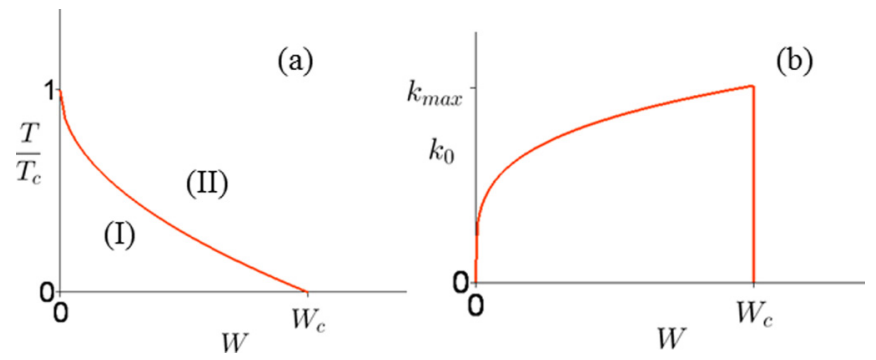

FIG. 1. (a) Phase diagram in diblock copolymers as a function of $W$. The red line displays the absolute instability temperature $\frac{T_{c}^{W}}{T_{c}}$ versus $W$. The value $W_{c}=\frac{1}{4}$ defines the limit of the ordered steady state. (b) The modulation wave vector $k_{0}$ versus $W$ in the ordered phase at $T=0$.

classical results of $[8,15]$. Then, the procedure is applied to chemically reactive mixtures and to phase separating binary mixtures under irradiation, both representing systems evolving under nonlocal external forces.

\section{A. Diblock copolymers}

In this system, the finite block length competes with the demixing of the constituent monomers, resulting in lamellar, hexagonal, or micellar structures [23] in 2D and gyroids, hexagonally packed cylinders, and bcc structure in 3D. In this case, $R \rightarrow \infty$ and $M, \Gamma$ are temperature independent. On the other hand, the decomposition of diblock copolymer is assumed to be a second-order phase transition. This last point implies that $\eta_{+}=-\eta_{-}$and then $\lambda=0$. Since this system is at thermodynamical equilibrium, the Lyapounov functional reduces to the classical free-energy functional [8]. $W$ is proportional to the inverse of the squared diblock copolymer size, is independent from the temperature, and $\hat{g}(k)=k^{-2}$ as already reported in the literature [8]. We also obtain the ordered and disordered phases as functions of the control parameter $W$ and temperature $T$, illustrated by the plots in Fig. 1(a). As expected, previous results from [8] are recovered. From Eq. (11), only one disordered and one ordered steady state can exist in the $(W, T)$ plane. The limit between these two steady states is defined by the line $T_{c}^{W}=T_{c}\left(1-2 W^{\frac{1}{2}}\right)$. For $T>T_{c}^{W}$, only a fully homogeneous disordered steady state exists [domain (II) in Fig. 1(a)]. For $T \leqslant T_{c}^{W}$, only one ordered steady state emerges [domain (I) in Fig. 1(a)] with a modulation wave vector $k_{0}=W^{\frac{1}{4}}$ [Fig. 1(b)] in agreement with previous calculations based on the calculation of the structure factor [14]. For the special case where $W=0$, the spinodal decomposition is recovered. The key point in this approach is to introduce naturally a critical value $W_{c}=\frac{1}{4}$. For $W>W_{c}$, Eqs. (11) and (12) have no solution and only the disordered phase (II) exists in the $(W, T)$ plane [Fig. 1(a)]. This analysis extends previous results obtained only for the $O(n)$ model [14] constraining the symmetry of $f(\eta(\mathbf{x}, t))$ and clearly points out that the disordered phase exists even below $W_{c}$ for nonzero temperature $T<T_{c}$.

\section{B. Chemically reactive mixtures}

In a binary reactive mixture, chemical reactions are responsible for the mixing of species. Assuming the decomposition
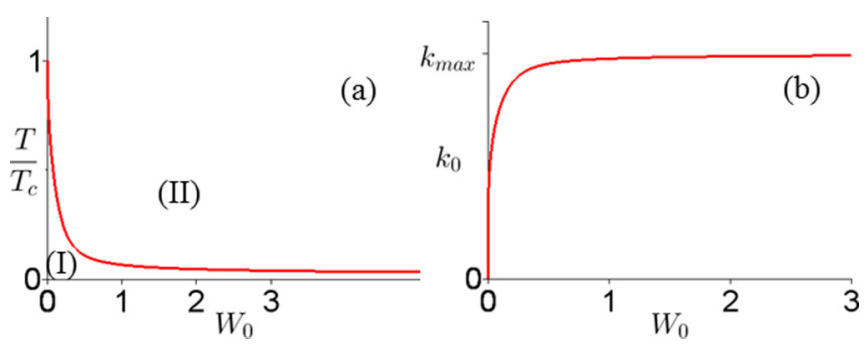

FIG. 2. (a) Phase diagram in chemical reactive mixtures as a function of $W_{0}$. The red line displays the absolute instability temperature $\frac{T_{c}^{W}}{T_{c}}$ versus $W_{0}$ defining the limit between ordered (I) and disordered (II) phases. (b) The modulation wave vector $k_{0}$ versus $W_{0}$ in the ordered phase calculated for $T=0\left(E_{0}=0.1\right)$.

to be a second-order phase transition, $T \eta_{+}=-\eta_{-}$and then $\lambda$ exhibits a null value. A steady-state pattern results from the balance between this effect and the demixing process associated with a spinodal decomposition [15]. Steady states in this forced system [3] are determined from the analysis of the Lyapunov functional. Given the Arrhenius dependence of the reaction rate $\Gamma, W(\tau)=W_{0} \exp \left(-\frac{E_{0}}{(1-\tau)}\right)$ is temperature dependent $\left(E_{0}=\frac{E_{a}}{k_{B} T_{c}}\right.$ is the reduced activation energy and $k_{B}$ is the Boltzmann constant). As is the case for diblock copolymers, $\hat{g}(k)=k^{-2}$ and the solutions of Eq. (11) show that only ordered (I) and disordered (II) phases exist. The critical temperature $T_{c}^{W}$ is then determined from the unique solution of the implicit equation $-\tau_{c}^{W}+2 W\left(\tau_{c}^{W}\right)^{\frac{1}{2}}=0$. As $T_{c}^{W}$ never vanishes, the ordered phase always exists and is no longer limited by a critical intensity level of the external force $W_{0}$.

Figure 2(a) displays an example of the $T_{c}^{W}$ line in the $\left(W_{0}, T\right)$ plane $\left(E_{0}=0.1\right)$. Solving Eqs. (11) and (12) yields the explicit dependence of the wave vector $k_{0}=W(\tau)^{\frac{1}{4}}=$ $W_{0}^{\frac{1}{4}} \exp \left(-\frac{E_{a}}{4 k_{B} T}\right)$ with the temperature $T$. For $T=0, k_{0}$ vanishes and only a spinodal decomposition occurs irrespective the values of $W_{0}$. For $T>0, k_{0}$ never vanishes, and one ordered steady state exists below $T_{c}^{W}$ with modulation wave vector $k_{0}$, Fig. 2(b).

\section{Immiscible alloys under irradiation}

In a $\mathrm{AB}$ binary alloy, irradiation induces mixing of species opposing the driving force of the spinodal decomposition. Thereby, the steady states produced differ from thermodynamical equilibrium states [24]. As already commented above, two control parameters $W$ and $R$ quantify the irradiation effects. $W$ relates to the efficiency of species mixing and is a function of the mass, the energy, the flux of impinging particles, and the density of the target [16]. $R$ is associated with the mean range of atomic displacements in the target triggered by incident particles impacts $[16,18]$. Both are assumed to be temperature independent. On the other hand, atomistic simulations indicate that $\hat{g}(k)=\frac{R^{2}}{1+k^{2} R^{2}}$ with a good approximation [16,25]. Solving Eqs. (11) and (12), a twofold solution is obtained:

$$
k_{0}(\tau)=0, \quad-\tau_{c}^{W}\left(1-3 \lambda^{2}\right)+W R^{2}=0,
$$




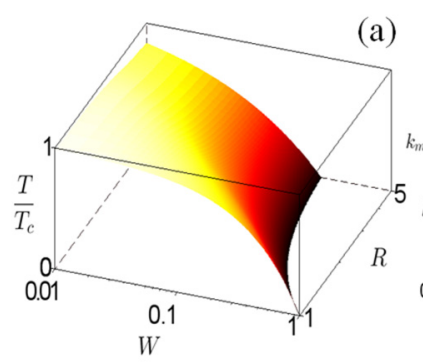

(a)
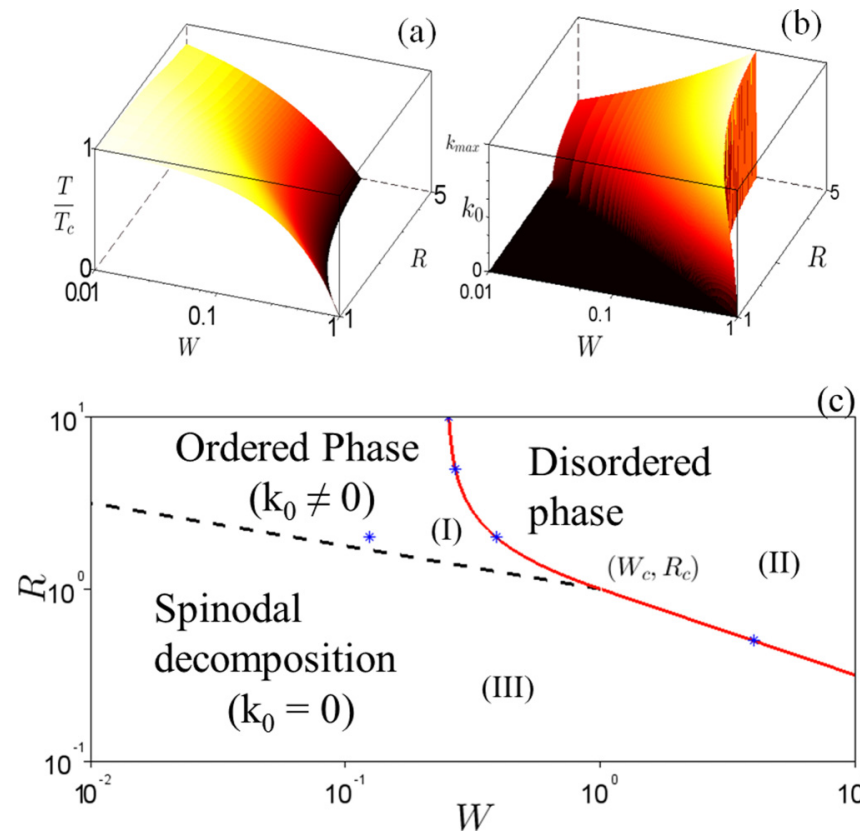

FIG. 3. Irradiation phase diagram in immiscible alloys. (a) Evolution of the absolute instability temperature surface $T_{c}^{W}$ as a function of $R, W$, and the temperature $T$. (b) Evolution of the modulation wave vector of the ordered phases versus $W$ and $R$ at $T=0$. (c) Limits of different domains computed for $T=0$ are drawn (red lines) as functions of $W$ and $R(\lambda=0$, see text) and compared with previous results [18] (blues stars). The dashed line displays the separation between the spinodal decomposition and the ordering process.

and

$$
\begin{aligned}
& k_{0}(\tau)=\left(W^{\frac{1}{2}}-R^{-2}\right)^{\frac{1}{2}}, \\
& -\tau_{c}^{W}\left(1-3 \lambda^{2}\right)+2 W^{\frac{1}{2}}-R^{-2}=0 .
\end{aligned}
$$

Solutions of Eqs. (13) and (14) determine not only the value of $T_{c}^{W}$, i.e., the limit between the ordered and disordered steady states, but also the number of ordered steady states below $T_{c}^{W}$. Figure 3(a) displays the variation of the surface $\frac{T_{c}^{W}}{T_{c}}$ as a function of $R$ and $W$ (for $\lambda=0$ ). Above this surface, $T>T_{c}^{W}$, only the fully disordered phase associated with a homogeneous distribution of $\mathrm{A}$ and $\mathrm{B}$ atoms exists. Below this surface, $T<T_{c}^{W}$, ordered steady states can be created under irradiation for nonvanishing $W$ values. The number of these ordered steady states and their modulation wave vectors $k_{0}$ are directly related to the function $\hat{g}(k)$. The two possible values for $k_{0}$ given by Eqs. (13) and (14) clearly appear in Fig. 3(b). For $W R^{4}<1, k_{0}=0$ is the unique solution and the spinodal decomposition occurs [black surface in Fig. 3(b)]. The associated steady state is given by a random distribution of precipitates in a homogeneous matrix. For
$W R^{4}>1, k_{0}=\left(W^{\frac{1}{2}}-R^{-2}\right)^{\frac{1}{2}}$ and the ordered steady-state results in a microstructure with precipitates of size proportional to $k_{0}^{-1}$. This analysis clearly implies that the demixion of the homogeneous disordered state under irradiation leads either to a spinodal decomposition $\left(k_{0}=0\right)$ or to an ordering process $\left(k_{0} \neq 0\right)$ in agreement with previous experimental results [26]. The surface $W R^{4}=1$ determines the partition between these two ordered steady states. Below $T_{c}^{W}$, irradiation disrupts coarsening during the spinodal decomposition enabling tailoring the microstructure with precipitate sizes proportional to $k_{0}^{-1}$. The different steady states and their limits are plotted versus $R$ and $W$ for $T=0$ in Fig. 3(c) and can be compared with previous results obtained for $T=0$ [18]. The $(W, R)$ plane can be split into three distinct areas: a disordered domain (II) and an ordered domain resulting either from a simple spinodal decomposition $\left(k_{0}=0\right)$ (III) or a modulated steady state, which does not exist in absence of irradiation (I). The phase limits (red line) between the homogeneous phase (II) and the ordered phases [(I) and (III)] defined, respectively, by $W=\left[\frac{1+R^{2}\left(1-3 \lambda^{2}\right)}{2 R^{2}}\right]^{2}$ and $W=\frac{1-3 \lambda^{2}}{R^{2}}$ are identical to previous results [18] (blue stars) confirming our analysis. The separation between the two ordered domains (I) and (III) defined by $W R^{4}=1$ (dashed black line) cannot be understood as a phase limit since precipitates exist in both phases. The point $\left(W_{c}, R_{c}\right)=\left(\left(1-3 \lambda^{2}\right)^{2},\left(1-3 \lambda^{2}\right)^{-\frac{1}{2}}\right)$ resulting from the intersections of the two ordered domains cannot be understood as a critical point as it has been previously reported in previous works [18].

\section{CONCLUSION}

In summary, this work presents a method to predict pattern formation in a demixing systems subject to nonlocal external forces, based on a Lyapounov functional that embodies their long-time evolution. Our method generalizes the approaches used for equilibrium systems with long-range order interactions [8]. The existence of the functional guaranties that no chaotic steady states nor limiting cycles can appear [1]. A clear physical interpretation of the nature and the number of steady states and their limits as a function of the control parameters and the temperature $T$ emerges from the analysis of the quadratic term of this Lyapounov functional. This approach allows us to determine the ordered steady states of various systems, in fair agreement with numerical simulations $[14,16,18]$ and experimental results $[3,27,28]$. The method is promising for microstructural engineering consisting in selecting the external force strength at a given temperature for fixing the amplitude of the modulation wave vector of the ordered phase as is needed by the application, e.g., optimization of the mechanical response. Finally, the method could help predicting ordered phases in systems under external forces such as superconducting, Langmuir, or epitaxial ferroelectric films processed by ion bombardment or ball milling.
[1] M. C. Cross and P. C. Hohenberg, Rev. Mod. Phys. 65, 851 (1993).
[2] A. G. Khatchaturyan, Theory of Structural Transformation in Solids (Wiley Interscience, New York, 1983). 
[3] M. Seul and D. Andelman, Science 267, 476 (1995).

[4] P. Tolédano and V. Dmitriev, Reconstructive Phase Transitions: In Crystals and Quasicrystals (World Scientific, Singapore, 1996).

[5] G. Lebon, D. Jou, and J. Casas-Vazquez, Understanding Nonequilibrium Thermodynamics: Foundations, Applications, Frontiers (Springer-Verlag, Berlin, 2008).

[6] M. Le Bellac, F. Mortessagne, and G. Batrouni, Equilibrium and Nonequilibrium Statistical Thermodynamics (Cambridge University Press, Cambridge, 2010).

[7] X. Bai, A. Voter, R. Hoagland, M. Nastasi, and B. Uberuaga, Science 327, 1631 (2010).

[8] T. Ohta and K. Kawasaki, Macromolecules 19, 2621 (1986).

[9] A. J. Bray, Adv. Phys. 43, 357 (1994).

[10] H. E. Cook, Acta Metall. 18, 297 (1970).

[11] L. Q. Chen and A. G. Khachaturyan, Phys. Rev. Lett. 70, 1477 (1993).

[12] L. Leibler, Macromolecules 13, 1602 (1980).

[13] S. Villain-Guillot and C. Josserand, Phys. Rev. E 66, 036308 (2002).

[14] S. C. Glotzer, D. Stauffer, and N. Jan, Phys. Rev. Lett. 72, 4109 (1994).

[15] S. C. Glotzer and A. Coniglio, Phys. Rev. E 50, 4241 (1994).

[16] D. Simeone, G. Demange, and L. Luneville, Phys. Rev. E 88, 032116 (2013).
[17] A. J. Bray and C. L. Emmott, Phys. Rev. B 52, R685 (1995).

[18] R. A. Enrique and P. Bellon, Phys. Rev. Lett. 84, 2885 (2000).

[19] S. C. Glotzer, E. A. Di Marzio, and M. Muthukumar, Phys. Rev. Lett. 74, 2034 (1995).

[20] D. Simeone and L. Luneville, Phys. Rev. E 81, 021115 (2010).

[21] G. Demange, Multiscale modeling of the microstructure of materials under irradiation within the PF framework, Ph.D. thesis, CentraleSupelec, 2015.

[22] This temperature can be understood as a critical temperature for a second order phase transition; it is related to temperatures associated with metastable phases for a first-order phase transition.

[23] Y. Gao, H. Deng, W. Li, F. Qiu, and A.-C. Shi, Phys. Rev. Lett. 116, 068304 (2016).

[24] P. Bellon and G. Martin, Phys. Rev. B 38, 2570 (1988).

[25] R. A. Enrique, K. Nordlund, R. S. Averback, and P. Bellon, J. Appl. Phys. 93, 2917 (2003).

[26] S. Chee, B. Stumphy, N. Vo, R. Averback, and P. Bellon, Acta Mater. 58, 4088 (2010).

[27] D. Simeone, G. Baldinozzi, D. Gosset, S. LeCaër, and L. Mazerolles, Phys. Rev. B 70, 134116 (2004).

[28] Y. Adda, M. Beyeler, and G. Brebec, Thin Solid Films 25, 107 (1975). 